\title{
TEST PROBLEMS FOR OPERATOR ALGEBRAS
}

\author{
EDWARD A. AZOFF
}

\begin{abstract}
Kaplansky's test problems, originally formulated for abelian groups, concern the relationship between isomorphism and direct sums. They provide a "reality check" for purported structure theories.

The present paper answers Kaplansky's problems in operator algebraic contexts including unitary equivalence of von Neumann algebras and equivalence of representations of (non self-adjoint) matrix algebras. In particular, it is shown that matrix algebras admitting similar ampliations are themselves similar.
\end{abstract}

\section{INTRODUCTION}

Kaplansky's test problems appear in [8]. They make sense in any category $C$ equipped with a binary "direct sum" operation $\oplus$; it does not really matter whether $\oplus$ has the naturality properties associated with products or coproducts [4]. It will be convenient to state the problems in the following form.

Test Problems. Suppose $a, b$, and $c$ are cbjects in $C$.

(P1) If $a$ and $b$ are isomorphic to direct summands of one another, must $a$ be isomorphic to $b$ ?

(P2) If $a \oplus a$ is isomorphic to $b \oplus b$, must $a$ be isomorphic to $b$ ?

(P3) Does isomorphism of $a \oplus c$ to $b \oplus c$ imply isomorphism of $a$ to $b$ ? If not, how should $c$ be restricted to validate this implication?

In [7], R. V. Kadison and I. M. Singer studied these problems in the context of unitary equivalence of Hilbert space operators. Their results illustrate the best one can hope for: affirmative answers to (P1) and (P2), and formulation of an appropriate finiteness condition on $c$ in response to (P3). Successful projects of this type have recently been carried out in the context of quasisimilarity of special classes of Hibert space operators: $C_{0}$ operators by $\mathrm{H}$. Bercovici [3] and bitriangular operators by K. Davidson and D. Herrero [5].

These papers illustrate two fundamentally different approaches to Kaplansky's problems. In [3] and [5], the authors develop canonical forms for the operators in question. No such forms were available to Kadison and Singer, who instead relied on the rich comparison theory for projections in von Neumann algebras. In Section 2 of the present paper, we review these ideas in various simple settings.

Received by the editors September 21, 1994.

1991 Mathematics Subject Classification. Primary 46L10, 16S50.

Key words and phrases. Ampliation, direct sum, unitary equivalence, similarity. 

bras.

In Section 3, we obtain the following results concerning von Neumann alge-

Theorem 1.1. Suppose $A$ and $B$ are von Neumann algebras whose 2-fold copies $A^{(2)}$ and $B^{(2)}$ are unitarily equivalent. Then $A$ is unitarily equivalent to $B$.

Theorem 1.2. Let $A, B$, and $C$ be von Neumann algebras of operators acting on arbitrary Hilbert spaces with $C$ not unitarily equivalent to a proper direct summand of itself. Then each of the following implies that $A$ is unitarily equivalent to $B$.

(1) $A$ and $B$ are unitarily equivalent to direct summands of one another.

(2) $A \oplus A$ is unitarily equivalent to $B \oplus B$.

(3) $A \oplus C$ is unitarily equivalent to $B \oplus C$.

The proof of 1.1 is accomplished by adapting Kadison and Singer's methods to the study of representations of a fixed von Neumann algebra. The proof of 1.2 , on the other hand, depends on studying a new equivalence relation on the central projections of a von Neumann algebra.

Sections 4 and 5 concern (not necessarily self-adjoint) operator algebras and are independent of Section 3.

Theorem 1.3. Suppose $A$ and $B$ are operator algebras whose commutants are finite-dimensional. If $A^{(2)}$ is similar to $B^{(2)}$ then $A$ is similar to $B$ as well.

Theorem 1.4. Let $A, B$, and $C$ be algebras of operators having finitedimensional commutants. Then each of the following implies that $A$ is similar to $B$.

(1) $A$ and $B$ are similar to direct summands of one another.

(2) $A \oplus A$ is similar to $B \oplus B$.

(3) $A \oplus C$ is similar to $B \oplus C$.

As in the von Neumann algebra setting, the relevant context for 1.3 is representations of a fixed algebra, while 1.4 addresses Kaplansky's problems in a category of operator algebras. This time, however, justification of 1.4 follows immediately from the fact that any operator algebra whose center is finitedimensional has a unique decomposition as a direct sum of factor algebras. The proof of 1.3, on the other hand, is accomplished by studying an appropriate notion of equivalence on the idempotents in a finite-dimensional algebra, where classical results of J. H. M. Wedderburn can be applied.

Throughout this paper, the term "direct sum" is assigned traditional operatortheoretic meanings. The direct sum of two vector spaces $V_{1}$ and $V_{2}$ is simply their cartesian product equipped with pointwise addition and scalar multiplication; if the original spaces have distinguished inner products, we define an inner product on $V_{1} \oplus V_{2}$ by setting

$$
\left\langle\left(x_{1}, x_{2}\right),\left(y_{1}, y_{2}\right)\right\rangle=\left\langle x_{1}, y_{1}\right\rangle+\left\langle x_{2}, y_{2}\right\rangle \text {. }
$$

The direct sum of two operators is defined componentwise, and if $A_{1}$ and $A_{2}$ are operator algebras acting on $V_{1}$ and $V_{2}$ respectively, then

$$
A_{1} \oplus A_{2}=\left\{a_{1} \oplus a_{2} \in L\left(V_{1} \oplus V_{2}\right): a_{1} \in A_{1}, a_{2} \in A_{2}\right\} .
$$


Finally, if $\pi_{1}$ and $\pi_{2}$ are representations of some common algebra $A$ on $V_{1}$ and $V_{2}$, then the direct sum representation is defined by

$$
\left(\pi_{1} \oplus \pi_{2}\right) a=\pi_{1}(a) \oplus \pi_{2}(a), \quad a \in A .
$$

To say that " $x$ is a direct summand of $y$ " means there is a $z$ for which $x \oplus z$ is (completely) isomorphic to $y$.

The language of category theory is avoided in the body of the paper, though we do identify the relevant object and morphism classes in an appendix.

While not required in the sequel, it should perhaps be mentioned that the motivation for 1.3 comes from the study of reflexive operator algebras [2]. An operator algebra $A$ is $k$-reflexive if its $k$-fold ampliation $A^{(k)}$ is reflexive. $k$ reflexivity implies $l$-reflexivity for $k \leq l$ and it seemed natural to introduce a fractional version of the notion, calling $A \frac{1}{2}$-reflexive if it is (similar to) a 2 -fold copy of some auxiliary reflexive algebra $B$. Theorem 1.3 lends credibility to this definition by implying that if $A$ is similar to a 2-fold copy of some other algebra $C$, then $C$ would have to be reflexive as well.

\section{SiNGLE OPERATORS}

The easiest operator-theoretic setting for Kaplansky's test problems involves unitary equivalence (i.e. isometric isomorphism) of the underlying Hilbert spaces.

Proposition 2.l. Let $H, J$, and $K$ be Hilbert spaces and suppose $K$ is not unitarily equivalent to a proper subspace of itself. Then each of the following implies that $H$ is unitarily equivalent to $K$.

(1) $H$ and $K$ are unitarily equivalent to subspaces of one another.

(2) $H \oplus H$ is unitarily equivalent to $J \oplus J$.

(3) $H \oplus K$ is unitarily equivalent to $J \oplus K$.

(4) (5)(6) The preceding conditions hold with "similar" in place of "unitarily equivalent".

Proof. (Orthogonal) dimension is a complete unitary invariant for Hilbert spaces. Since $\operatorname{dim} K$ is finite by hypothesis, (1), (2), and (3) reduce to cardinal arithmetic. As for (4), (5), and (6), polar decomposition shows that similar Hilbert spaces are automatically unitarily equivalent.

The ease of the preceding proof reflects the fact that Hilbert space dimension is a very satisfying invariant. The next proposition illustrates the corresponding utility of Jordan Canonical Forms.

Proposition 2.2. Let $a, b$, and $c$ be operators acting on finite-dimensional complex vector spaces. Then each of the following implies that $a$ is similar to $b$.

(1) $a$ and $b$ are similar to a direct summands of one another.

(2) $a \oplus a$ is similar to $b \oplus b$.

(3) $a \oplus c$ is similar to $b \oplus c$.

Proof. For each polynomial $p$ and operator $a$, set $n_{p}(a)$ equal to the rank of $p(a)$. Then $\left\{n_{p}\right\}$ is a complete set of similarity invariants since $n_{p}(a)=n_{p}(b)$ for all $p$ implies that $a$ and $b$ have a common Jordan Canonical Form. Since $n_{p}(a \oplus c)=n_{p}(a)+n_{p}(c)$, the proof reduces to integer arithmetic. 
Theorem 2.3. Let $a, b$, and $c$ be operators acting on arbitrary Hilbert spaces and suppose that $c$ is not unitarily equivalent to a direct summand of itself. Then each of the following implies that $a$ is unitarily equivalent to $b$.

(1) $a$ and $b$ are unitarily equivalent to direct summands of one another.

(2) $a \oplus a$ is unitarily equivalent to $b \oplus b$.

(3) $a \oplus c$ is unitarily equivalent to $b \oplus c$.

When the underlying Hilbert spaces are finite dimensional, 2.3 can be proved in the same style as 2.2. For each polynomial $p$ in two non-commuting variables and each operator $a$, set $t_{p}(a)$ equal to the trace of $p\left(a, a^{*}\right)$. It is a theorem of W. Specht [11] that the $\left\{t_{p}(a)\right\}$ form a complete set of unitary invariantsnote in particular that $t_{1}(a)$ recovers the dimension of the underlying Hilbert space. (C. Pearcy [10] proved that only finitely many of the $\left\{t_{p}\right\}$ are needed to distinguish between unitary equivalence classes in $M_{n}(\mathbb{C})$ for any fixed $n$.) The observation that each $t_{p}$ is additive (i.e. $\left.t_{p}(a \oplus b)=t_{p}(a)+t_{p}(b)\right)$ thus reduces the proof to integer arithmetic.

On the other hand, there is no hope of finding a complete set of "computable" unitary invariants for operators on infinite-dimensional spaces. (More precisely, the associated quotient Borel structure is not countably separated. See W. Arveson [1] and J. Ernest [6] for discussion of this phenomenon.) We do, however, have a rich comparison theory for projections in von Neumann algebras, which tells us a lot about the structure of single operators. This is the tool exploited by Kadison and Ringrose in their proof of 2.3 (under a stronger assumption for 2.3.3).

Direct sums and ampliations are usually thought of as external constructions. The first step in the proof of Theorem 2.3 is an internal reformulation of its hypotheses and conclusions.

Definition 2.4. Let $a \in L(H, K)$ and suppose $p \in L(H)$ is a (self-adjoint) projection. Then $a_{p}$ denotes the restriction of $a$ to the range of $p$. If $A$ is a subset of $L(H, K)$, we write $A_{p}$ for $\left\{a_{p}: a \in A\right\}$.

Proposition 2.5. Let $a$ be a (bounded Hilbert space) operator and write $A$ for the von Neumann algebra of all operators which commute with it and its adjoint. Suppose $p$ and $q$ are projections in $A$. Then the following statements imply one another.

(1) $a_{p}$ is unitarily equivalent to $a_{q}$.

(2) $p$ and $q$ are von Neumann equivalent projections in $A$.

Proof. (1) $\Longrightarrow$ (2) Given $u: \operatorname{ran} p \rightarrow \operatorname{ran} q$ with $u a_{p}=a_{q} u$, take $v$ to be the extension of $u$ to $H$ which vanishes on $\operatorname{ker} p$. Then $v$ is a partial isometry in $A$ having the range of $p$ as its initial space and the range of $q$ as its final space.

(2) $\Longrightarrow$ (1) Given $v \in A$ with $v^{*} v=p$ and $v v^{*}=q$, take $u$ to be its restriction to ran $p$.

Proposition 2.6. Let $a$ and $b$ be unitarily equivalent operatois, acting on possibly different Hilbert spaces, say $u a u^{*}=b$. Suppose $p$ is a projection commuting with $a$ and set $q=u p u^{*}$. Then

(1) $q$ commutes with $b$,

(2) $a_{p}, a_{1-p}$ are unitarily equivalent to $b_{q}, b_{1-q}$ respectively. 
Proof. It is easy to check that $q$ and $b$ commute. From the equation $u p=q u$, we conclude that $u$ maps the range of $p$ onto the range of $q$, so that $u_{p}$ is a unitary operator between $\operatorname{ran} p$ and $\operatorname{ran} q$. From the equation $u a=b u$, we therefore learn that $u_{p} a_{p}=b_{q} u_{p}$, which establishes the unitary equivalence of $a_{p}$ and $b_{q}$. The proof is completed by replacing $p$ and $q$ by their complements $1-p$ and $1-q$.

Proof of Theorem 2.3. (1) By hypothesis, there are projections $p, r$ commuting with $a, b$ respectively such that $a \simeq b_{r}$ and $b \simeq a_{p}$. Applying Proposition 2.6, we also find a projection $q$ with $b_{r} \simeq\left(a_{p}\right)_{q}=a_{p q}$. Thus $a \simeq a_{p q}$ by the transitivity of unitary equivalence. Proposition 2.5 tells us that the identity 1 and $p q$ are equivalent projections in the ${ }^{*}$-commutant $A$ of $a$ : Since $1 \leq$ $p \leq p q$, we conclude that 1 and $p$ are equivalent as well, whence a second application of 2.5 tells us that $a=a_{1} \simeq a_{p} \simeq b$.

(2) Fix an operator $c$ unitarily equivalent to $a \oplus a$ (and hence also to $b \oplus$ $b$ ), and write $C$ for the ${ }^{*}$-commutant of $c$. Applying 2.6 to the canonical projection $1 \oplus 0$ associated with $a \oplus a$, we find a projection $p \in C$ with $c_{p} \simeq a \simeq c_{1-p}$. Similarly, we get $q \in C$ with $c_{q} \simeq b \simeq c_{1-q}$. Applying 2.5, we conclude that $p \sim 1-p$ and $q \sim 1-q$. A basic result in comparison theory (Proposition 8 on page 49 of [13]) yields $p \sim q$, whence a second application of 2.5 tells us that $a \simeq c_{p} \simeq c_{q} \simeq b$.

(3) Fix $d$ unitarily equivalent to $a \oplus c$ and $b \oplus c$ and write $D$ for its *-commutant. Apply 2.6 to get projections $p, q \in D$ with $d_{p} \simeq c, d_{1-p} \simeq$ $a, d_{q} \simeq c$, and $d_{1-q} \simeq b$. Thus $p \sim q$ by 2.5. The hypothesis that $c$ is not unitarily equivalent to a direct summand of itself means that $p$ and $q$ are finite projections. Proposition 1.38 on page 304 of [12] then tells us that $1-p \sim 1-q$, so $a \simeq b$ by 2.5 .

\section{Von Neumann Algebras}

Definition 3.1. Let $p, q$ be projections in the commutant of a von Neumann algebra $A$.

(1) $A_{p}=\left\{a_{p}: a \in A\right\}$.

(2) $\pi_{p}: A \rightarrow A_{p}$ by $\pi_{p}(a)=a_{p}$.

(3) $A_{p} \simeq A_{q}$ if there is a unitary operator $u: \operatorname{ran} p \rightarrow \operatorname{ran} q$ such that $u A_{p}=A_{q} u$ (as sets).

(4) $\pi_{p} \simeq \pi_{q}$ if there is a unitary operator $u: \operatorname{ran} p \rightarrow \operatorname{ran} q$ such that $u a_{p}=a_{q} u$ for all $a \in A$.

Proposition 3.2. Let $p, q$ be projections in the commutant of a von Neumann algebra $A$. Then the following statements imply one another.

(1) $\pi_{p} \simeq \pi_{q}$.

(2) $p$ and $q$ are equivalent projections relative to the von Neumann algebra $A^{\prime}$.

Proof. (1) $\Longrightarrow$ (2) Given $u: \operatorname{ran} p \rightarrow \operatorname{ran} q$ with $u a_{p}=a_{q} u$ for all $a \in A$, take $v$ to be the extension of $u$ to $H$ which vanishes on ker $p$. Then $v$ is a partial isometry in $A^{\prime}$ having the range of $p$ as its initial space and the range of $q$ as its final space.

(2) $\Longrightarrow$ (1) Given $v \in A^{\prime}$ with $v^{*} v=p$ and $v v^{*}=q$, take $u$ to be its restriction to ran $p$. 
Proposition 3.3. Let $A \subset L(H)$ and $B \subset L(K)$ be unitarily equivalent von Neumann algebras, say $u A u^{*}=B$. Define $\phi: L(H) \rightarrow L(K)$ by $\phi(t)=u t u^{*}$. Suppose $p$ and $q$ are projections in $A^{\prime}$. Then

(1) $\phi(p) \in B^{\prime}$,

(2) $A_{p} \simeq B_{\phi(p)}$,

(3) $p \sim q$ relative to $A^{\prime}$ iff $\phi(p) \sim \phi(q)$ relative to $B^{\prime}$.

Proof. It is easy to check that $\phi(p)$ lies in the commutant of $B$. From the equation $u p=\phi(p) u$, we conclude that $u$ maps the range of $p$ onto the range of $\phi(p)$, so that $u_{p}$ is a unitary operator between ran $p$ and $\operatorname{ran} \phi(p)$. From the equation $u A=B u$, we therefore learn that $u_{p} A_{p}=B_{\phi(p)} u_{p}$, which establishes the unitary equivalence of $A_{p}$ and $B_{\phi(p)}$. The proof is completed by noting that if $v \in A^{\prime}$ implements the equivalence between $p$ and $q$, then $\phi(v) \in B^{\prime}$ implements an equivalence between $\phi(p)$ and $\phi(q)$

Proof of Theorem 1.1. Let $C$ be a von Neumann algebra unitarily equivalent to $A^{(2)}$ and $B^{(2)}$. The restriction of $A^{(2)}$ to the range of the canonical projection $1 \oplus 0$ is unitarily equivalent to $A$, and relative to the commutant of $A^{(2)}$, this projection is equivalent to its complement. Applying Propositions 3.2 and 3.3, we therefore find a projection $p$ in $C^{\prime}$ with $C_{p} \simeq A$ and $p \sim 1-p$ relative to $C^{\prime}$. Similarly, we get a projection $q$ with $C_{q} \simeq B$ and $q \sim 1-q$ relative to $C^{\prime}$. Comparison theory tells us that $p \sim q$ relative to $C^{\prime}$ whence $A \simeq B$.

To see the difference between Theorems 1.1 and 1.2, note that the canonical projection $p \equiv 1 \oplus 0$ commutes with $A \oplus A$, and the restrictions of $A \oplus A$ to $p$ and $1-p$ are both unitarily equivalent to $A$, but the corresponding representations $\pi_{p}$ and $\pi_{1-p}$ are not equivalent, i.e., the operator implementing the unitary equivalence between $(A \oplus A)_{p}$ and $(A \oplus A)_{1-p}$ has nothing to do with the commutant of $A \oplus A$.

Definition 3.4. Let $p$ and $q$ be central projections in a von Neumann algebra $A$. We say that $p$ is (centrally) equivalent to $q$ if there is a unitary operator $u: \operatorname{ran} p \rightarrow \operatorname{ran} q$ such that $u A_{p} u^{*}=A_{q}$ (as sets). We write $p \approx q$ if $p$ is centrally equivalent to $q$ and $p \ll q$ if $p$ is centrally equivalent to some $r \leq q$.

Theorem 3.5. Let $p$ and $q$ be central projections in the von Neumann algebra A.

(1) If $p \ll q$ and $q \ll p$, then $p \approx q$.

(2) If $p \approx 1-p$ and $q \approx 1-q$, then $p \approx q$.

(3) If $p \approx q$, then either $p$ is centrally equivalent to a proper subprojection of itself or $1-p \approx 1-q$.

Postponing the proof of 3.5 for a moment, we first apply it to Theorem 1.2. The internalization we need is already contained in Propositions 3.2 and 3.3.

Proof of Theorem 1.2. (1) By hypothesis, there are projections $p, r$ in the centers of $A, B$ respectively such that $A \simeq B_{r}$ and $B \simeq A_{p}$. Applying Proposition 3.3, we also find a central projection $q$ with $B_{r} \simeq\left(A_{p}\right)_{q}=A_{p q}$. Thus $A \simeq A_{p q}$ by the transitivity of unitary equivalence. By definition, $1 \approx p q$, and since $1 \leq p \leq p q$, we conclude from 3.5.1 that $1 \approx p$, whence $A=A_{1} \simeq A_{p} \simeq B$. 
(2) Fix a von Neumann algebra $C$ unitarily equivalent to $A \oplus A$ (and hence also to $B \oplus B)$. Applying 3.3.2 to the central projection $1 \oplus 0$ associated with $A \oplus A$, we find a projection $p$ in the center of $C$ with $C_{p} \simeq A \simeq C_{1-p}$. Similarly, we get $q$ in the center of $C$ with $C_{q} \simeq B \simeq C_{1-q}$. By definition, $p \approx 1-p$ and $q \approx 1-q$, so $p \approx q$ by 3.5.2, whence $A \simeq C_{p} \simeq C_{q} \simeq B$.

(3) Fix $D$ unitarily equivalent to $A \oplus C$ and $B \oplus C$. Apply 3.3.2 to get central projections $p, q \in D$ with $D_{p} \simeq C, D_{1-p} \simeq A, D_{q} \simeq C$, and $D_{1-q} \simeq B$. Thus $p \approx q$. The hypothesis that $C$ is not unitarily equivalent to a direct summand of itself means that $p$ is not centrally equivalent to a proper subprojection of itself, so $1-p \approx 1-q$ by 3.5 .3 whence $A \simeq B$.

It remains to prove 3.5. For the most part, we follow the format of the corresponding proofs for von Neumann equivalence as presented on pages 291304 of [12]. For the remainder of this section, the von Neumann algebra $A$ will be fixed, all projections discussed will be central projections in $A$, and equivalence will refer to the relation introduced in Definition 3.4.

Definition 3.6. A partial isometry $v$ is admissible if $v^{*} v$ and $v v^{*}$ are central projections in $A$ and $v A=A v$ as sets. The projection $z$ is hypercentral if 0 is the only subprojection of $z$ which is equivalent to a subprojection of $1-z$.

Lemma 3.7. Let $p$ and $q$ be central projections in $A$.

(1) $p \approx q$ iff there is an admissible partial isometry having the range of $p$ as its initial space and the range of $q$ as its final space.

(2) If $\left\{p_{i}: i \in I\right\}$ and $\left\{q_{i}: i \in I\right\}$ are orthogonal families of central projections in $A$ with $p_{i} \approx q_{i}$ for each $i$, then $\sum p_{i} \approx \sum q_{i}$.

(3) If $p \approx q$ and $z$ is hypercentral, then $z p \approx z q$.

(4) If 0 is the only subprojection of $p$ which is equivalent to a subprojection of $q$, then there is a hypercentral projection $z$ with $p \leq z$ and $q \leq 1-z$.

Proof. (1) Given $u: \operatorname{ran} p \rightarrow \operatorname{ran} q$ with $u A_{p}=A_{q} u$, take $v$ to be the extension of $u$ to the entire underlying Hilbert space which vanishes on $(\operatorname{ran} p)^{\perp}$. Given $a, b \in A$ with $u a_{p}=b_{q} u$, the fact that $p$ and $q$ commute with $A$ implies that $v a=b v$; thus $v A=A v$.

Conversely, given a partial isometry $v$ having ran $p$ as initial space and ran $q$ as final space with $v A=A v$, take $u$ to be the restriction of $v$ to ran $p$. Then given $a, b \in A$ with $v a=b v$, we also have $u a_{p}=b_{q} u$; thus $u A_{p}=A_{q} u$.

(2) Choose admissible partial isometries $\left\{v_{i}: i \in I\right\}$ implementing the hypothesized equivalences. It is clear that the series $\sum v_{i}$ converges strongly to a partial isometry $w$ with the desired initial and final space. To check that $w$ is admissible, let $a \in A$ be given. For each $i, p_{i} a$ also belongs to $A$ so there is a $b_{i} \in A$ with $v_{i} p_{i} a=b_{i} v_{i}=b_{i} q_{i} v_{i}$. Since $\left\|b_{i} q_{i}\right\|=\left\|p_{i} a\right\|$ for each $i$ and $v_{i} v_{j}=0$ for $i \neq j$, we see that the series $\sum b_{i} q_{i}$ converges to an element $b \in A$ with $w a=b w$. Thus $w A \subset A w$ and $w A=A w$ by symmetry.

(3) Choose $v$ with $v^{*} v=p$ and $v v^{*}=q$ and take $w=v z$. Then $w^{*} w=$ $z p z=z p$. That $w w^{*} \leq z$ follows since $z$ is hypercentral while $w w^{*}=$ $v z v^{*} \leq v v^{*}=q$ only depends on the fact that $z$ is a projection. Thus $w w^{*} \leq$ $z q$. On the other hand, $q=v z v^{*}+v(1-z) v^{*} \leq z q+(1-z) q=q$ forces $w w^{*}=z q$.

(4) Extend $\{p\}$ to a maximal orthogonal family $\mathscr{F}$ of projections which are equivalent to subprojections of $p$. Take $z$ to be the supremum of $\mathscr{F}$. The maximality of $\mathscr{F}$ shows that $z$ is hypercentral. 
Proof of Theorem 3.5.1. Choose admissible partial isometries $u$ and $v$ with

$$
u^{*} u=p, \quad u u^{*}=q_{1} \leq q, \quad v^{*} v=q, \quad \text { and } \quad v v^{*}=f_{1} \leq p .
$$

Write $p_{0}=p$ and $q_{0}=q$. Construct two decreasing sequences of projections inductively by the formulas $p_{n+1}=v q_{n} v^{*}$ and $q_{n+1}=u p_{n} u^{*}$. Also, set $p_{\infty}=$ $\lim p_{n}$ and $q_{\infty}=\lim q_{n}$. For each $n, u\left(p_{2 n}-p_{2 n+1}\right)$ is an admissible partial isometry whose initial space is the range of $p_{2 n}-p_{2 n+1}$ and whose final space is the range of $q_{2 n+1}-q_{2 n+2}$. Thus $p_{2 n}-p_{2 n+1} \approx q_{2 n+1}-q_{2 n+2}$ and a similar argument shows that $q_{2 n}-q_{2 n+1} \approx p_{2 n+1}-p_{2 n+2}$. Since $q_{\infty}=u p_{\infty} u^{*}$, we also have $p_{\infty} \approx q_{\infty}$. Thus

$$
\begin{aligned}
p & =\sum_{n=0}^{\infty}\left(p_{2 n}-p_{2 n+1}\right)+\sum_{n=0}^{\infty}\left(p_{2 n+1}-p_{2 n+2}\right)+p_{\infty} \\
& \approx \sum_{n=0}^{\infty}\left(q_{2 n+1}-q_{2 n+2}\right)+\sum_{n=0}^{\infty}\left(q_{2 n}-q_{2 n+1}\right)+q_{\infty}=q .
\end{aligned}
$$

Proposition 3.8. (Comparability) Given central projections $p$ and $q$ in $A$, there is a hypercentral projection $z$ satisfying $z p \ll z q$ and $(1-z) p \gg(1-z) q$.

Proof. Choose maximal orthogonal families of central projections $\left\{p_{i}: i \in I\right\}$ and $\left\{q_{i}: i \in I\right\}$ in $A$ satisfying $p_{i} \leq p, q_{i} \leq q$, and $p_{i} \approx q_{i}$ for each $i \in I$. Set $p_{0}=\sum_{i \in I} p_{i}$ and $q_{0}=\sum_{i \in I} q_{i}$. By maximality and Lemma 3.7.4, there is a hypercentral projection $z$ with $p-p_{0} \leq 1-z$ and $q-q_{0} \leq z$. Applying Lemma 3.7.3, we therefore get $z p=z p_{0} \approx z q_{0} \leq z q$ and $(1-z) q=(1-z) q_{0} \approx$ $(1-z) p_{0} \leq(1-z) p$.

Proof of 3.5.2. Apply the Comparison Theorem to the projections $p(1-q)$ and $q(1-p)$ to get a hypercentral $z$ satisfying $z p(1-q) \ll z q(1-p)$ and $(1-z) p(1-q) \gg(1-z) q(1-p)$. Adding $z p q$ to the first "inequality" and $(1-z)(1-p)(1-q)$ to the second yields

$$
z p \ll z q \text { and }(1-z)(1-q) \gg(1-z)(1-p) .
$$

Applying the hypothesis and Lemma 3.7.3, the last inequality yields

$$
(1-z) p \approx(1-z)(1-p) \ll(1-z)(1-q) \approx(1-z) q .
$$

Thus $z p \ll z q$ and $(1-z) p \ll(1-z) q$ whence $p \ll q$ and the desired conclusion $p \approx q$ follows by symmetry.

Proposition 3.9. Suppose $e$ is an infinite central projection in $A$. Then there is a projection $f \leq e$ and a hypercentral projection $z$ in $A$ such that $z e$ is infinite and $z f \approx z(e-f) \approx z e$.

Proof. By assumption, there is an admissible partial isometry $u$ satisfying $u^{*} u=e$ and $u u^{*}<e$. Set $p_{n}=u^{n}\left(e-u u^{*}\right)\left(u^{*}\right)^{n}$ for each non-negative integer $n$. Note that the $\left\{p_{n}\right\}$ are mutually orthogonal and equivalent, and $p_{n} \leq e$ for each $\mathrm{n}$. Let $\left\{q_{i}: i \in I\right\}$ be a maximal family of orthogonal equivalent projections majorized by $e$ which contains the original family $\left\{p_{n}\right\}$. Write $q_{0}=e-\sum_{i \in I} q_{i}$. Choose a hypercentral projection $z$ satisfying $z q_{0} \ll z p_{0}$ and $(1-z) q_{0} \gg(1-z) p_{0}$. By maximality, we know that $p_{0}$ cannot be equivalent to 
a subprojection of $q_{0}$ so the last inequality shows that $z e>z p_{0}>0$. Express $I$ as the disjoint union of two sets $I_{1}$ and $I_{2}$ of equal (infinite) cardinality . Then

$$
z e=z q_{0}+\sum_{i \in I_{1}} z q_{i}+\sum_{i \in I_{2}} z q_{i} \ll z q_{0}+\sum_{i \in I_{1}} z q_{i} \ll \sum_{i \in I_{2}} z q_{i} \ll z e .
$$

Thus $f=q_{0}+\sum_{i \in I_{1}} q_{i}$ satisfies $z f \approx z(e-f) \approx z e$. If $z e$ were finite, we would have $z f=z(e-f)=z e$ which is impossible since we already know $z e>0$.

Proposition 3.10. If $p$ and $q$ are finite projections then $p \vee q$ is also finite.

Proof. Since we are dealing with central projections, $p \vee q=p+q(1-p)$. Thus, replacing $q$ by $q(1-p)$ if necessary, we can assume that $p q=0$. Suppose for purposes of contradiction that $e=p+q$ is infinite and apply Proposition 3.9 to get $f$ and $z$. Next, apply Proposition 3.8 to get another hypercentral projection $w$ satisfying $w z p f \ll w z q(e-f)$ and $(1-w) z p f \gg(1-w) z q(e-f)$. Now

$$
\begin{gathered}
w z e \approx w z f=w z p f+w z q f \ll w z q(e-f)+w z q f=w z q \text { and } \\
(1-w) z e \approx(1-w) z(e-f)=(1-w) z p(e-f)+(1-w) z q(e-f) \ll(1-w) z p .
\end{gathered}
$$

It follows that $w z e$ and $(1-w) z e$ are finite. Since $w$ is hypercentral, we reach the conclusion that $z e$ is finite in contradiction of Proposition 3.9.

Proof of Theorem 3.5.3. Suppose $p \approx q$ with $p$ finite. Write $e=p \vee q$ which we have just seen is finite. Apply comparability to get a hypercentral $z$ with $z(e-p) \approx h \leq z(e-q)$ and $(1-z)(e-q) \approx k \leq(1-z)(e-p)$. Now $z e=z p+z(e-p) \approx z q+h \leq z e$ whence the finiteness of $z e$ implies that $h=z(e-q)$ i.e. $z(e-p) \approx z(e-q)$. Similarly,

$$
(1-z) e=(1-z) q+(1-z)(e-q) \approx(1-z) p+k \leq(1-z)(e-p)
$$

whence $(1-z)(e-p) \approx(1-z)(e-q)$. Adding equivalences yields $e-p \approx e-q$ whence $1-p \approx 1-q$.

\section{COMPARISON THEORY FOR IDEMPOTENTS IN FINITE-DIMENSIONAL ALGEBRAS}

Throughout this section, $A$ will denote a finite-dimensional complex algebra with identity.

Definition 4.1. Two idempotents $e, f$ in $A$ are equivalent (denoted $e \sim f$ ) if there exist $x, y \in A$ satisfying $x y=e$ and $y x=f$.

The goal of this section is the following proposition.

Proposition 4.2. Let $e, f$ be idempotents in $A$.

(1) If $e \sim 1-e$ and $f \sim 1-f$ then $e \sim f$.

(2) If $e \sim f$, then $1-e \sim 1-f$.

Example 4.3. Take $A=M_{n}$, the algebra of linear transformations on $\mathbb{C}_{n}$. Then $e \sim f$ iff rank $e=\operatorname{rank} f$ iff $e$ is similar to $f$. Thus Proposition 4.2 holds for $A=M_{n}$. 
Proof. Suppose first that $e \sim f$, and choose $x, y$ as in the definition. Then $e=$ $x(y x) y=x f y$ so rank $e \leq \operatorname{rank} f$, whence rank $e=\operatorname{rank} f$ by symmetry.

Next, suppose rank $e=\operatorname{rank} f$. There is a $z$ in $M_{n}$ which maps range $e$ onto range $f$ and ker $e$ onto ker $f$, and it is easy to check that $z e z^{-1}=f$.

To complete the cycle, suppose $z e z^{-1}=f$. Then $x=e z^{-1}$ and $y=z e$ implement the equivalence $e \sim f$.

Having established that $e \sim f$ iff rank $e=\operatorname{rank} f$, Proposition 4.2 becomes a matter of arithmetic. For example, if $e \sim f$, then $\operatorname{rank}(1-e)=n-\operatorname{rank} e$ $e=n-\operatorname{rank} f=\operatorname{rank}(1-f)$, whence $1-e \sim 1-f$.

Proposition 4.4. Let $e, f, z$ be idempotents in $A$ with $z$ lying in the center of $A$. Then $e \sim f$ iff $e z \sim f z$ and $e(1-z) \sim f(1-z)$.

Proof. Given $x, y$ which implement the equivalence between $e$ and $f$, it is easy to check that $x z, y z$ implement the equivalence between $e z$ and $f z$, so $e z \sim f z$ and $e(1-z) \sim f(1-z)$. Conversely, given $s, t$ which implement the equivalence between $e z, f z$ and $u, v$ which implement the equivalence $e(1-z) \sim f(1-z)$, note that $s z+u(1-z)$ and $t z+v(1-z)$ implement the equivalence $e \sim f$.

We next use Wedderburn's structure theory to prove Proposition 4.2 for semisimple algebras. We recall the relevant definitions here, but refer the reader to [9] for details.

Definition 4.5. Let $A$ be a finite-dimensional algebra over $\mathbb{C}$ with identity.

(1) The radical of $A$ is the intersection of its maximal (two-sided) ideals. It is denoted $R(A)$.

(2) $A$ is simple if it has no non-trivial two-sided ideals.

(3) $A$ is semi-simple if its radical is $\{0\}$.

Proposition 4.6. Proposition 4.2 holds if $A$ is semi-simple.

Proof. The class of algebras satisfying Proposition 4.2 is obviously closed under algebraic isomorphism; Proposition 4.4 implies that it is also closed under the taking of direct sums. By the Wedderburn Structure Theorems, we know that $A$ is isomorphic to a direct sum of full matrix algebras, whence the proof is completed by appealing to Example 4.3.

Let $\pi: A \rightarrow A / R(A)$ be the canonical projection. It is clear that $A / R(A)$ is semi-simple. The next result says that equivalences in $A / R(A)$ can be lifted to equivalences in $A$. We follow the development on pages 124-126 of [9].

\section{Proposition 4.7.}

(1) Let $e, f$ be orthogonal idempotents in $A / R(A)$ and suppose $u$ is an idempotent in $A$ with $\pi(u)=e$. Then there is an idempotent $v$ in $A$ with $v u=u v=0$ and $\pi(v)=f$.

(2) Let $u, v$ be idempotents in $A$ such that $\pi(u)$ and $\pi(v)$ are equivalent. Then $u$ and $v$ are equivalent.

Proof. (1) Choose $x \in A$ with $\pi(x)=f$ and set $y=(1-u) x(1-u)$. We still have $\pi(y)=f$. In particular, $\pi\left(y^{2}-y\right)=0$ so $y^{2}-y$ is nilpotent and thus the spectrum of $y$ is contained in $\{0,1\}$. Let $\phi$ be the characteristic function of $\{z \in \mathbb{C}:|z-1|<1 / 2\}$. Then $\phi$ is analytic in a neighborhood of the spectrum 
of $y$ and $\phi(y)$ is idempotent. Since we are working in a finite-dimensional algebra, we can find a polynomial $p$ with $p(0)=0$ and $p(1)=1$ such that $\phi(y)=p(y)$. Setting $v=p(y)$, we note that $v^{2}=v$ and $\pi(v)=p(\pi(y))=f$. That $u v=v u=0$ follows since $p$ has no constant term.

(2) Choose $x, y \in A$ with $x y-u \in R(A)$ and $y x-v \in R(A)$. Replacing $x, y$ by $u x v$ and $v y u$ respectively if necessary, we may assume $x=$ $u x v$ and $y=v y u$. Set $z=y(1+x y-u)^{-1}$. Since $u$ commutes with $x y$, it also commutes with $(1+x y-u)^{-1}$ whence $z=v z=z u$. Also $x z=$ $u(1+x y-u)(1+x y-u)^{-1}=u$, while $(z x)^{2}=z(x z) x=z u x=z x$ so that $z x$ is an idempotent commuting with $v$. It follows that $v-z x$ is idempotent. On the other hand, $\pi(v-z x)=0$, so $v-z x$ is nilpotent. The conclusion is that $v=z x$ and we have already seen that $x z=u$.

Proof of Proposition 4.2. (1) We have $\pi(e) \sim 1-\pi(e)$ and $\pi(f) \sim 1-\pi(f)$. Since $\pi(A)$ is semi-simple, we get $\pi(e) \sim \pi(f)$ by 4.6. But then $e \sim f$ by 4.7.1.

(2) From $\pi(e) \sim \pi(f)$ we get $1-\pi(e) \sim 1-\pi(f)$ by 4.6 whence $1-e \sim 1-f$ by 4.7.2.

\section{Algebras With Finite-Dimensional commutants}

Throughout this section, the term "operator algebra" will refer to an algebra of linear transformations acting on a complex vector space whose commutant is finite-dimensional.

Definition 5.1. Let $e, f$ be idempotents in the commutant of an operator algebra $A$.

(1) $A_{e}=\left\{a_{e} \mid a \in A\right\}$.

(2) $\pi_{e}: A \rightarrow A_{e}$ by $\pi_{e}(a)=a_{e}$.

(3) $A_{e} \simeq A_{f}$ if there is a surjective $u: \operatorname{ran} e \rightarrow \operatorname{ran} f$ with $u A_{e}=A_{f} u$ (as sets).

(4) $\pi_{e} \simeq \pi_{f}$ if there is a surjective $u: \operatorname{ran} e \rightarrow \operatorname{ran} f$ with $u a_{e}=a_{f} u$ for all $a \in A$.

Proposition 5.2. Let $e, f$ be idempotents in the commutant of an operator algebra $A \subset L(V)$. Then the following statements imply one another.

(1) $\pi_{e} \simeq \pi_{f}$.

(2) $e$ and $f$ are equivalent relative to the relation on the idempotents in the operator algebra $A^{\prime}$ studied in the preceding section.

Proof. (1) $\Longrightarrow$ (2) Given $u: \operatorname{ran} e \rightarrow \operatorname{ran} f$ with $u a_{e}=a_{f} u$ for all $a \in A$, take $y$ to be the extension of $u$ to $V$ which vanishes on ker $e$ and take $x$ to be the extension of $u^{-1}$ to $V$ which vanishes on ker $f$. Then $x$ and $y$ commute with $A, x y=e$, and $y x=f$.

(2) $\Longrightarrow$ (1) Given $x, y \in A^{\prime}$ with $x y=e$ and $y x=f$, take $u$ to be the restriction of $y$ to ran $e$.

Proposition 5.3. Let $A \subset L(V)$ and $B \subset L(W)$ be similar operator algebras, say $u A u^{-1}=B$. Define $\phi: L(V) \rightarrow L(W)$ by $\phi(t)=u t u^{-1}$. Suppose $e$ and $f$ are idempotents in $A^{\prime}$. Then

(1) $\phi(e) \in B^{\prime}$. 
(2) $A_{e} \simeq B_{\phi(e)}$.

(3) $e \sim f$ relative to $A^{\prime}$ iff $\phi(e) \sim \phi(f)$ relative to $B^{\prime}$.

Proof. It is easy to check that $\phi(e)$ and $b$ commute. From the equation $u e=$ $\phi(e) u$, we conclude that $u$ maps the range of $e$ onto the range of $\phi(e)$, so that $u_{e}$ is an isomorphism between $\operatorname{ran} e$ and $\operatorname{ran} \phi(e)$. From the equation $u A=$ $B u$, we therefore learn that $u_{e} A_{e}=B_{\phi(e)} u_{e}$, which establishes the similarity of $A_{e}$ and $B_{\phi(e)}$. The proof is completed by noting that if $x, y \in A^{\prime}$ implement an equivalence between $e$ and $f$, then $\phi(x), \phi(y) \in B^{\prime}$ implement an equivalence between $\phi(e)$ and $\phi(f)$.

Proof of Theorem 1.3. Let $C$ be an operator algebra similar to $A^{(2)}$ and $B^{(2)}$. The restriction of $A^{(2)}$ to the range of the canonical idempotent $1 \oplus 0$ is similar to $A$, and relative to the commutant of $A^{(2)}$, this idempotent is equivalent to its complement. Applying Propositions 5.2 and 5.3, we therefore find an idempotent $e \in C^{\prime}$ with $C_{e} \simeq A$ and $e \sim 1-e$ relative to $C^{\prime}$. Similarly, we get an idempotent $f$ with $C_{f} \simeq B$ and $f \sim 1-f$ relative to $C^{\prime}$. Proposition 4.2 tells us that $e \sim f$ relative to $C^{\prime}$ whence $A \simeq B$.

Proof of Theorem 1.4. Note that every operator algebra whose center is finitedimensional can be "uniquely" expressed as a direct sum of factors; isomorphisms between such algebras merely permute the factors. (In other words, the proof of 3.5 is trivial when the center of $A$ is finite-dimensional.)

\section{APPENDIX: RELEVANT CATEGORIES}

Proposition 2.1, Parts (1) (2) (3). The objects are Hilbert spaces. The morphisms are partial isometries.

Note that if $u \in L(H, K)$ and $v \in L(K, H)$ are partial isometries satisfying $u v=I_{K}$, then $u$ must be onto and $v$ must be one-to-one. In particular, $u$ is an isomorphism in this category if and if it is unitary.

Proposition 2.1, Parts (4) (5) (6). The objects are Hilbert spaces. The morphisms are bounded operators.

The isomorphisms in this category are the invertible operators.

Proposition 2.2. The objects are linear transformations on finite-dimensional complex vector spaces. The morphisms are intertwining operators, that is given $a \in L(H)$ and $b \in L(K)$, we take hom $(a, b)=\{x \in L(H, K): x a=b x\}$.

Theorem 2.3. The objects are Hilbert space operators. The morphisms are intertwining partial isometries.

Theorem 1.1. The objects are weak*-continuous representations of a fixed abstract $W^{*}$-algebra (that is a $C^{*}$-algebra which is a dual Banach space). The morphisms are intertwining operators, that is, given representations $\pi: A \rightarrow$ $L(H)$ and $\sigma: A \rightarrow L(K)$, an admissible morphism between $\pi$ and $\sigma$ is a partial isometry $u \in L(H, K)$ satisfying $u \pi(a)=\sigma(a) u$ for each $a \in A$.

Theorem 1.2. The objects are concrete von Neumann algebras. The morphisms are setwise intertwining partial isometries, that is, given $A \subset L(H)$ and $B \subset$ $L(K)$, we take $\operatorname{hom}(A, B)=\{u \in L(H, K): u$ is a partial isometry and $u A=B u$ as sets $\}$. 
Theorem 1.3. The objects are representations of a fixed abstract algebra whose center is finite-dimensional. The morphisms are intertwining operators, that is, given representations $\pi: A \rightarrow L(V)$ and $\sigma: A \rightarrow L(W)$, an admissible morphism between $\pi$ and $\sigma$ is a transformation $x \in L(H, K)$ satisfying $x \pi(a)=\sigma(a) x$ for each $a \in A$

Theorem 1.4. The objects are operator algebras having finite-dimensional commutants. The morphisms are setwise intertwining operators, that is, given $A \subset L(V)$ and $B \subset L(W)$, we take $\operatorname{hom}(A, B)=\{x \in L(V, W): x A=B x$ (as sets) $\}$.

\section{REFERENCES}

1. W.B. Arveson, An invitation to $C^{*}$-algebras, Springer-Verlag, New York, 1976.

2. E.A. Azoff, On finite rank operators and preannihilators, Mem. Amer. Math. Soc. 357 (1986).

3. H. Bercovici, Three test problems for quasisimilarity, Canad. J. Math. 39 (1987), 880-892.

4. I. Bucur and A Deleanu, Introduction to the theory of categories and functors, Wiley, New York, 1968.

5. K.R. Davidson and D.A. Herrero, The Jordan form of a bitriangular operator, J. Funct. Anal. 94 (1990), 27-73.

6. J. Ernest, Charting the operator terrain, Mem. Amer. Math. Soc. 171 (1976).

7. R.V. Kadison and I.M. Singer, Three test problems in operator theory, Pacific J. Math. 7 (1957), 1101-1106.

8. I. Kaplansky, Infinite abelian groups, 2nd ed., The University of Michigan Press, Ann Arbor, 1965.

9. __ Fields and rings, 2nd ed., The University of Chicago Press, Chicago, 1972.

10. C. M. Pearcy, A complete set of unitary invariants for operators generating finite $W^{*}$ algebras of type I, Pacific J. Math. 12 (1962), 1405-1416.

11. W. Specht, Zur Theorie der Matrizen II, Jber. Deutsch. Math. Verein. 50 (1940), 19-23.

12. M. Takesaki, Theory of operator algebras I, Springer-Verlag, New York, 1979.

13. D.M. Topping, Lectures on von Neumann algebras, Van Nostrand Reinhold, New York, 1971.

Department of Mathematics, University of Georgia, Athens, Georgia 30602-7403

E-mail address: azof $f @ a l p h a . m a t h . u g a . e d u$ 